\title{
Correction to: The Italian version of the Juvenile Arthritis Multidimensional Assessment Report (JAMAR)
}

\author{
Alessandro Consolaro ${ }^{1,2} \cdot$ Francesca Bovis $^{1} \cdot$ Angela Pistorio $^{3} \cdot$ Rolando Cimaz $^{4} \cdot$ Fabrizio De Benedetti $^{5}$. \\ Angela Miniaci ${ }^{6} \cdot$ Fabrizia Corona $^{7} \cdot$ Valeria Gerloni $^{8} \cdot$ Silvana Martino $^{9} \cdot$ Serena Pastore $^{10} \cdot$ Patrizia Barone $^{11}$. \\ Sara Pieropan ${ }^{12} \cdot$ Elisabetta Cortis $^{13} \cdot$ Rosa Anna Podda $^{14} \cdot$ Romina Gallizzi $^{15} \cdot$ Adele Civino $^{16}$. Francesco La Torre ${ }^{17}$. \\ Donato Rigante $^{18} \cdot$ Rita Consolini $^{19} \cdot$ Maria Cristina Maggio $^{20} \cdot$ Silvia Magni-Manzoni $^{5} \cdot$ Francesca Perfetti $^{5}$. \\ Giovanni Filocamo $^{7}$. Claudia Toppino ${ }^{9}$ - Francesco Licciardi ${ }^{9}$. Marco Garrone ${ }^{1}$ - Silvia Scala ${ }^{1}$. Elisa Patrone ${ }^{1}$.

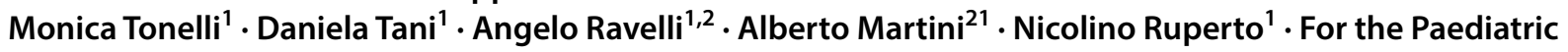 \\ Rheumatology International Trials Organisation (PRINTO)
}

Published online: 21 May 2018

๑) Springer-Verlag GmbH Germany, part of Springer Nature 2018

\section{Correction to:}

Rheumatology International (2018) 38 (Suppl 1):S251-S258

https://doi.org/10.1007/s00296-018-3960-1

The family name of author Francesco La Torre was incorrect in the published article. The correct family name should read as La Torre F.

The original article can be found online at https://doi.org/10.1007/ s00296-018-3960-1.

Alessandro Consolaro

alessandroconsolaro@gaslini.org

$\triangle$ Nicolino Ruperto

nicolaruperto@gaslini.org

Extended author information available on the last page of the article 


\section{Affiliations}

\section{Alessandro Consolaro ${ }^{1,2} \cdot$ Francesca Bovis $^{1} \cdot$ Angela Pistorio $^{3} \cdot$ Rolando Cimaz $^{4} \cdot$ Fabrizio De Benedetti ${ }^{5}$. Angela Miniaci ${ }^{6} \cdot$ Fabrizia Corona $^{7} \cdot$ Valeria Gerloni $^{8}$. Silvana Martino ${ }^{9}$. Serena Pastore ${ }^{10}$. Patrizia Barone ${ }^{11}$. Sara Pieropan ${ }^{12}$. Elisabetta Cortis ${ }^{13} \cdot$ Rosa Anna Podda ${ }^{14} \cdot$ Romina Gallizzi $^{15} \cdot$ Adele Civino $^{16} \cdot$ Francesco La Torre $^{17}$. Donato Rigante ${ }^{18} \cdot$ Rita Consolini $^{19} \cdot$ Maria Cristina Maggio $^{20} \cdot$ Silvia Magni-Manzoni $^{5}$. Francesca Perfetti ${ }^{5}$. Giovanni Filocamo $^{7}$. Claudia Toppino ${ }^{9}$. Francesco Licciardi ${ }^{9} \cdot$ Marco Garrone $^{1}$. Silvia Scala ${ }^{1}$. Elisa Patrone ${ }^{1}$.

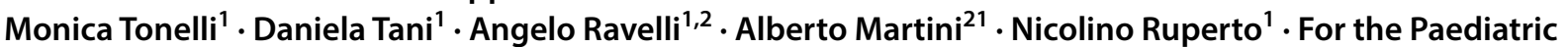 Rheumatology International Trials Organisation (PRINTO)}

Francesca Bovis

francescabovis@gaslini.org

Angela Pistorio

angelapistorio@gaslini.org

Rolando Cimaz

r.cimaz@meyer.it

Fabrizio De Benedetti

fabrizio.debenedetti@opbg.net

Angela Miniaci

angela.miniaci@aosp.bo.it

Fabrizia Corona

fcorona@policlinico.mi.it

Valeria Gerloni

valeria_gerloni@yahoo.it

Silvana Martino

silvana.martino@unito.it

Serena Pastore

serena.pastore@burlo.trieste.it

Patrizia Barone

barone@policlinico.unict.it

Sara Pieropan

sara.pieropan@aovr.veneto.it

Elisabetta Cortis

elisabetta.cortis@uslumbria2.it

Rosa Anna Podda

rpodda@unica.it

Romina Gallizzi

rgallizzi@unime.it

Adele Civino

adelecivino@gmail.com

Francesco La Torre

latorre_francesco@virgilio.it

Donato Rigante

drigante@gmail.com

Rita Consolini

rita.consolini@med.unipi.it

Maria Cristina Maggio

sbenfratello@libero.it

Silvia Magni-Manzoni

silvia.magnimanzoni@opbg.net

Francesca Perfetti

francesca.perfetti81@gmail.com
Giovanni Filocamo

giovanni.filocamo@gmail.com

Claudia Toppino

claudia.toppino@gmail.com

Francesco Licciardi

francesco.licciardi@gmail.com

Marco Garrone

marcogarrone@gaslini.org

Silvia Scala

silviascala@gaslini.org

Elisa Patrone

elisapatrone@gaslini.org

Monica Tonelli

tonelli_m@libero.it

Daniela Tani

danielatani@gaslini.org

Angelo Ravelli

angeloravelli@gaslini.org

Alberto Martini

albertomartini@gaslini.org

1 Clinica Pediatrica e Reumatologia, Paediatric Rheumatology International Trials Organisation (PRINTO), Istituto Giannina Gaslini, Via Gaslini 5, 16147 Genoa, Italy

2 Dipartimento di Pediatria, Università di Genova, Genoa, Italy

3 Servizio di Epidemiologia e Biostatistica, Istituto Giannina Gaslini, Genoa, Italy

4 Dipartimento di Pediatria, Azienda Ospedaliero-Universitaria Meyer, Florence, Italy

5 Division of Rheumatology, IRCCS Ospedale Pediatrico Bambino Gesù, Rome, Italy

6 Salute della Donna, del Bambino e dell'Adolescente-Padiglione 16 Ambulatorio di reumatologia, Azienda Ospedaliero-Universitaria S.Orsola-Malpighi, Bologna, Italy

7 Clinica Pediatrica II De Marchi, Fondazione IRCCS Ca' Granda-Ospedale Maggiore Policlinico, Milan, Italy

8 Divisione di Reumatologia, Istituto Gaetano Pini, Milan, Italy

9 Clinica Pediatrica, Paediatrics Departments, Università di Torino, Turin, Italy

10 Scienze della Riproduzione e dello Sviluppo, IRCCS Burlo Garofolo, Trieste, Italy 
11 Clinica Pediatrica, Azienda Policlinico Università di Catania, Catania, Italy

12 Reumatologia, Policlinico G.B.Rossi, Verona, Italy

13 Struttura Complessa Pediatria, Ospedale Santa Maria Della Stella, Località Ciconia-Orvieto, Terni, Italy

14 Clinica e Biologia dell'età evolutiva, Ospedale Regionale Microcitemia-II Clinica Pediatrica, Cagliari, Italy

15 Department of Human Pathology in Adulthood and Childhood, University of Messina, Messina, Italy

16 Pediatria, Azienda Ospedaliera Cardinale G. Panico, Tricase, LE, Italy
17 Centro Regionale-HUB-di Reumatologia Pediatrica, Ospedale Antonio Perrino, Brindisi, Italy

18 Pediatria, Università Cattolica Sacro Cuore, Roma, Italy

19 Pediatria, Ospedale Santa Chiara, Università di Pisa, Pisa, Italy

20 University Department Pro.Sa.M.I. “G. D’Alessandro”, University of Palermo, Palermo, Italy

21 Direzione Scientifica, Istituto Giannina Gaslini, Via Gaslini 5, 16147 Genoa, Italy 